\title{
Assessing Non-Technical Skills in Iranian Orthopedic Surgeons: An Observational Study
}

\author{
Mehdi Hasanshahi 1(D), Reza Kalantari 2*iD), Zahra Zamanian 2 iD, \\ Somayeh Gheysari ${ }^{1}$ (D), Ehsan Bakhshi ${ }^{3}$ (D)
}

1. Dept. of Operating Room, Faculty of Nursing, Shiraz University of Medical Sciences, Shiraz, Iran

2. Dept. of Ergonomics, Faculty of Public Health, Shiraz University of Medical Sciences, Shiraz, Iran

3. Health Center, Islamabad-e-Gharb Health Network, Kermanshah University of Medical Sciences, Kermanshah, Iran

\begin{tabular}{|c|c|}
\hline Article Info & ABSTRACT \\
\hline d 0 10.30699/jambs.28.129.191 & $\begin{array}{l}\text { Background \& Objective: Non-technical skills are critical for patient safety and } \\
\text { good surgical outcomes. Thus, surgeons should master these skills. This study }\end{array}$ \\
\hline Received: 2020/03/30; & \\
\hline $\begin{array}{l}\text { Accepted: 2020/07/15; } \\
\text { Published Online: } 31 \text { July 2020; }\end{array}$ & $\begin{array}{l}\text { Materials \& Methods: This cross-sectional study was conducted in two orthopedic } \\
\text { surgery wards in 2019. A total of } 22 \text { orthopedic surgeons were observed in this survey. }\end{array}$ \\
\hline Use your device to scan and read the & $\begin{array}{l}\text { The data were collected using Non-Technical Skills for Surgeons (NOTSS) through } \\
\text { observing and recording events during surgeries. }\end{array}$ \\
\hline 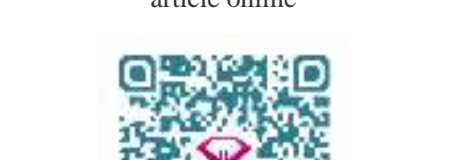 & $\begin{array}{l}\text { Results: "Leadership" was the best non-technical skill with score of } 3.14 \text { out of } 4 \text {, } \\
\text { while "communication and teamwork" had the lowest score ( } 3.02 \text { out of } 4) \text {. There was } \\
\text { a significant relationship between work experience and "projecting and anticipating } \\
\text { future state" }(P=0.009) \text {. }\end{array}$ \\
\hline 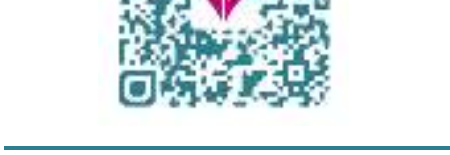 & $\begin{array}{l}\text { Conclusion: The studied orthopedic surgeons showed acceptable non-technical } \\
\text { skills. However, poor behaviors were observed in some domains. Educational } \\
\text { programs, policymaking interventions, and supervision can improve the surgeons' } \\
\text { non-technical skills. }\end{array}$ \\
\hline $\begin{array}{l}\text { Corresponding Information: } \\
\text { Reza Kalantari, } \\
\text { Dept. of Ergonomics, Faculty of } \\
\text { Public Health, Shiraz University of } \\
\text { Medical Sciences, Shiraz, Iran } \\
\text { E-Mail: rkalantari@ sums.ac.ir }\end{array}$ & $\begin{array}{l}\text { Keywords: Human factors, Non-technical skills, Orthopedic surgery, Operating } \\
\text { room, Surgeon }\end{array}$ \\
\hline $\begin{array}{l}\text { Copyright } \odot 2020 \text {, This is an } \\
\text { copy and redistribution of the }\end{array}$ & $\begin{array}{l}\text { ess article distributed under the terms of the Creative Commons Attribution-noncommercial } 4.0 \text { International License which permits } \\
\text { oncommercial usages with proper citation. }\end{array}$ \\
\hline
\end{tabular}

\section{Introduction}

Operating rooms are the most common places of a healthcare system in which adverse events occur (1). One main cause of these events is weakness in non-technical skills (2), which have been defined as cognitive and social skills that improve healthcare staff's performance (3). It has been mentioned that non-technical skills are protective against human errors and their consequences (4) and can complement technical skills (3). Defects in non-technical skills such as teamwork, leadership, decision-making, communication, and situational awareness are not rare in operating rooms (5) and can lead to poor performance (6). Inappropriate communications can take place in operating rooms and jeopardize patient safety (7). A previous study indicated that poor communication in the operating room contributed to $43 \%$ of surgical errors (8). Based on the Scottish audit of surgical mortality, failures in non-technical skills such as decision-making were found to be the main contributor to surgical deaths (9). Therefore, investigation of non-technical skills during surgeries is particularly important.

During a surgical procedure, the surgeon is mainly responsible for controlling all activities in the operating room (10). Modern surgery no longer depends completely on the surgeons' technical abilities (11) and surgeons need both technical and non-technical skills (12). In fact, being a good surgeon is more than having a good pair of hands. Surgeons should be active, communicate with other team members, and use their potentials during surgeries (11). Surgeons' non-technical skills are also important for patient safety (13). Some expert surgeons have shown sufficient non-technical skills as an inseparable part of their behaviors (14). These skills represent the surgeons' cognitive (15) and interacting competencies (16).

Previous studies on surgeons' non-technical skills have shown contradictory results. In the research 
performed by Greenberg et al. (2007), surgeons were the most involved team members in communication failures (13). Kalantari et al. (2019) found that surgeons had poor non-technical skills post-operatively (17). In another study, surgeons were the best team members in communication and teamwork domains (18). Rao et al. reported proper non-technical skills among surgeons (19). Behaviors such as asking anesthetist for update, talking about the progress of the operation, and following surgery protocols were considered among positive behaviors that could be observed in good surgeons' performance (20).

Adverse events, such as wrong site surgeries, are more prevalent in orthopedic surgeries than other types of surgery (21). Furthermore, Iranian surgeons' nontechnical skills have not been assessed previously. As non-technical skills can prevent adverse events and improve surgical outcomes, this study is aimed to assess non-technical skills among Iranian orthopedic surgeons.

\section{Materials and Methods}

This cross-sectional study was conducted at two public hospitals in Tehran, Iran in 2019. One surgical ward (orthopedic) and one procedure (hand surgery) were selected using simple random sampling method. This was done to make the surgeons' scores more comparable. All the orthopedic surgeons working in the hospitals were invited to participate in the study. The inclusion criterion was willingness to take part in the research. Out of all 24 surgeons, 22 agreed to participate. The surgeons' demographic information was provided by the hospital administrators.

Non-Technical Skills for Surgeons (NOTSS) that is a behavioral marker system designed to assess surgeons' non-technical skills during surgeries (20), was used in this study. This tool was developed by Yule et al. in the University of Aberdeen, Scotland in 2006 to evaluate surgeons' non-technical skills during the surgical process (16). NOTSS provides a specific classification for identifying surgeons' behaviors involving in a successful surgery (22). NOTSS is a gold standard for surgeons' non-technical skills training (23); it is also a valid and reliable tool (24), that has shown good usability in different cultures. This observational assessment tool contains four behavioral domains developed based on behavioral markers as follows: "decision-making", "situational awareness", "leadership", and "communication and teamwork". The definitions of the categories (20) have been presented in Table 1.

Table 1. The definition and elements of each category in the NOTSS

\begin{tabular}{|c|c|c|}
\hline Category & Definition & Elements \\
\hline $\begin{array}{l}\text { Situational } \\
\text { awareness }\end{array}$ & $\begin{array}{l}\text { "Having dynamic awareness from the situation during surgery } \\
\text { and the data received from the environment, understanding their } \\
\text { meaning and thinking about what may happen next." }\end{array}$ & $\begin{array}{l}\text { 1. Gathering information } \\
\text { 2. Understanding information } \\
\text { 3. Projecting and anticipating the future state. }\end{array}$ \\
\hline Decision-making & $\begin{array}{l}\text { "Skills needed for diagnosing the situation and judging for } \\
\text { selecting an appropriate action." }\end{array}$ & $\begin{array}{l}\text { 1. Selecting and communicating options } \\
\text { 2. Implementing and reviewing decisions } \\
\text { 3. Considering options. }\end{array}$ \\
\hline $\begin{array}{l}\text { Communication } \\
\text { and teamwork }\end{array}$ & $\begin{array}{l}\text { "Necessary skills for working in a team context to ensure that } \\
\text { the surgical team has an acceptable shared picture of the } \\
\text { situation and can complete tasks effectively." }\end{array}$ & $\begin{array}{l}\text { 1. Exchanging information } \\
\text { 2. Establishing a shared understanding } \\
\text { 3. Coordinating team activities. }\end{array}$ \\
\hline Leadership & $\begin{array}{l}\text { "Leading the team and providing directions, demonstrating } \\
\text { high standards of clinical practice and care, and being } \\
\text { considerate of the needs of the team members." }\end{array}$ & $\begin{array}{l}\text { 1. Setting and maintaining standards } \\
\text { 2. Supporting others } \\
\text { 3. Coping with pressure. }\end{array}$ \\
\hline
\end{tabular}

Each category includes three elements, which could be assessed based on a list of good and poor behaviors. A 1-4 scoring system has been designed to evaluate the elements showing poor, marginal, standard, and good levels of non-technical skills. Furthermore, there is a "not applicable" option for the cases in which a skill is not required or is irrelevant.

In order to prepare the NOTSS to use in Iranian hospitals, forward-backward translation was used. Doing so, first the tool was independently translated into Persian by two bilingual experts in psychology and surgery. Then, two different translators conducted the back translation. Finally, a coordinator provided the final version of the translated tool. The face validity of the instrument was first checked by seven experts in human factors and seven experienced surgeons. Content validity of the translated tool was also assessed using Content Validity Index (CVI) and Content Validity Ratio (CVR) by 15 surgeons (CVI=0.86 and $\mathrm{CVR}=0.59$ ). Moreover, inter-observer reliability of the Persian version of the NOTSS was tested comparing the results of the observations of two independent observers; i.e., a human factors expert and an experienced operating room technician who were trained for using the tool. In this context, ten hand surgeries were randomly selected and observed. Intraclass correlation coefficient for two observers was 0.88 , which showed the good inter-observer reliability of the tool. The results of Cronbach's alpha showed internal consistency among the four categories of the 
instrument. This value was more than 0.7 for all four skill categories $(0.841,0.877,0.902$, and 0.857 for situational awareness, decision-making, communication and teamwork, and leadership, respectively). Fit indices were also at the acceptable level. Finally, the construct validity of the translated tool was confirmed using confirmatory factor analysis.

The trained human factors specialist dressed the operating room uniform and attended the operating room before the surgical team members. He introduced himself to the team members and asked for their permission for data collection. He stayed in a place that could see all the team interactions and behaviors without disturbing the surgical team members. The observer assessed the surgeons' non-technical skills using the Persian version of NOTSS based on positive and negative behavioral examples. After observing and recording the surgical events, each element of the four categories was scored separately. Then, the score of each category was calculated based on the mean scores of the elements.

Descriptive and inferential statistics were used to analyze the data. The normality of the variables was assessed using Shapiro-Wilks test. Mean and standard deviation were employed to compute the mean scores of NOTSS. In addition, Pearson's correlation coefficient was used to assess the relationship between the scores and the participants' age and work

Table 2. The demographics of the studied surgeons

\begin{tabular}{cc} 
Demographics & Description \\
\hline Age & $53.36 \pm 9.17$ years (range: $38-66$ ) \\
\hline Work experience & $16.81 \pm 7.76$ years (range: $6-28)$ \\
Marital status & Married: 20, single: 2 \\
Gender & Male: 20 , Female: 2 \\
\hline
\end{tabular}

Table 3. Means and standard Mean of the four categories and the related elements of non-technical skills

\begin{tabular}{|c|c|}
\hline Category of non-technical skills & Score (out of 4) \\
\hline Situational awareness & $3.04 \pm 0.42$ \\
\hline Gathering information & $3.04 \pm 0.84$ \\
\hline Understanding information & $2.90 \pm 0.52$ \\
\hline Projecting and anticipating the future state & $3.13 \pm 0.46$ \\
\hline Decision-making & $3.05 \pm 0.40$ \\
\hline Selecting and communicating options & $3.18 \pm 0.50$ \\
\hline Implementing and reviewing decisions & $2.90 \pm 0.61$ \\
\hline Considering options & $3.09 \pm 0.52$ \\
\hline Communication and teamwork & $3.02 \pm 0.52$ \\
\hline Exchanging information & $3.31 \pm 0.40$ \\
\hline Establishing a shared understanding & $2.95 \pm 0.57$ \\
\hline Coordinating team activities & $2.86 \pm 0.77$ \\
\hline Leadership & $3.14 \pm 0.43$ \\
\hline Setting and maintaining standards & $3.18 \pm 0.58$ \\
\hline Supporting others & $2.90 \pm 0.68$ \\
\hline Coping with pressure & $3.31 \pm 0.56$ \\
\hline
\end{tabular}

experience. Independent sample t-test was also used to evaluate the relationship between the NOTSS scores and other demographic features. All data analyses were carried out in SPSS 22 (SPSS Inc., Chicago, IL., USA) and $\mathrm{P}$-value $<0.05$ was considered to be statistically significant.

Procedures followed were in accordance with the ethical standards of the responsible committee on human experimentation and with Helsinki Declaration of 1975, as revised in 1983. The Ethics Committee of Tehran University of Medical Sciences approved this study.

\section{Results}

This study was conducted on 22 surgeons. The demographics of the study population is illustrated in Table 2 .

The mean score of non-technical skills for surgeons was 12.27 \pm 1.48 out of 16 (range: 9.98-14.65). Additionally, the mean scores of the four skill categories were $3.04 \pm 0.42$ (situational awareness), $3.05 \pm 0.40 \quad$ (decision-making), $\quad 3.02 \pm 0.52$ (communication and teamwork), and 3.14 \pm 0.43 (leadership). Positive correlation was observed among the scores of the categories. The mean scores of the elements are separately presented in Table 3 . 
The married and male surgeons obtained higher scores. The results revealed no significant relationship and not even a specific trend between the total score of NOTSS and age and work experience. However, significant correlation was observed between age and "projecting and anticipating the future state" $(P=0.01$, Pearson's correlation coefficient $=0.5)$, age and "establishing a shared understanding" $(P=0.02$, Pearson's correlation coefficient $=-0.493)$, and work experience and "projecting and anticipating the future state" $\quad(P=0.009, \quad$ Pearson's correlation coefficient $=0.545)$.

\section{Discussion}

This study aimed to assess the Iranian orthopedic surgeons' non-technical skills at two public hospitals. The results showed that the surgeons' non-technical skills were at the acceptable level, while there were some deficits in certain behavioral domains.

There is a need for valid and reliable tools to assess non-technical skills in surgeries. NOTSS was used in the present study because it is the most inclusive tool to assess surgeons' non-technical skills and a specialized tool in comparison to others. In this study, the psychometric properties of the Persian version of NOTSS were confirmed. This result was consistent with those of the former studies performed on NOTSS $(25,26)$. The tool has been translated into some other languages, accepted by surgeons in many countries, and registered as a standard instrument for surgeons (4). NOTSS is considered to approximately be a complete tool, which covers many non-technical skills behaviors very well, and can be useful in finding the training needs.

The current study results showed that the scores of the non-technical skills categories were at the acceptable level (mean score > 3); but, there was a need for improvement in some behavioral domains. This result was in agreement with the results of some former studies. For instance, Wauben et al. reported a good level of non-technical skills among surgeons (27). Mills et al. also concluded that surgeons had appropriate safety culture, teamwork, and communication (28). In another investigation, the surgeons had a positive perspective towards nontechnical skills and enjoyed working in a team structure (29). However, the scores of all non-technical skills elements were higher than those reported in the study carried out by Tsuburaya et al. (30). This could be attributed to the cultural and educational differences between the studied populations.

In the present study, "leadership" was ranked as the highest skill, while "decision-making", "situational awareness", and "communication and teamwork" were rated approximately the same. Similarly, Kalantari et al. reported that surgeons had better leadership scores in comparison to other non-technical skills (17). This could result from the higher level of hierarchy in Iran's hospitals, which gives more authority to surgeons. Team members usually know the surgeon as the leader of the surgical team, which could be the reason of the higher score of the "leadership" category. This skill is necessary for efficient team performance (31). Surgeons have more authority compared to other team members and, consequently, their leadership behaviors are more visible during surgeries (32). Indeed, it seems that the surgeons were slightly better in personal skills compared to team-related ones. The order of nontechnical skills scores in the current study was different from the study performed by Tsuburaya et al., which ranked situational awareness as the best skill (30). Situational awareness is very essential because other non-technical skills might not succeed in its absence (31). Failures in teamwork and communication could cause many preoperative complications, as well $(6,13)$.

The present findings revealed no significant relationship between NOTSS total score and demographics. Dedy et al. also found no significant relationship between the surgeons' demographics and non-technical skills (33). Nonetheless, results of the present study indicated a number of significant relationships at the level of non-technical elements. For instance, older and more experienced surgeons anticipated the future state significantly better than the younger ones; but, they were significantly weaker in "establishing a shared understanding".

Although the mean score of each skill category was acceptable, concerning gestures were recorded in some surgeries that could negatively impact the scores of NOTSS categories. The surgeon arriving late to the operating room, leaving before the surgery ends, and neglecting other team members occurred in many operation theatres. Delay in the operating room can cause disaccord among surgical team members, while presence and coordination of the surgeon in the primary stages of surgery can establish a positive atmosphere and help better performance. Kalantari et al. assessed surgical teams using the Observational Teamwork Assessment for Surgery (OTAS) and rated the surgical team's skills as "moderate" (17). Other studies reported a reduction in the surgeons' levels of non-technical skills during the last steps of the surgical procedure $(17,27)$. Leaving the operating room during the surgical procedure is an inappropriate action that is prevalent among almost all surgeons. This can have a negative impact on performance as well as patient safety. Furthermore, the scores of some behavioral elements were lower than the acceptable level, which could be a sign of discrepancy in the surgeons' understanding of non-technical skills.

Former studies have shown that interventions could be useful in improvement of non-technical skills. For instance, Tsuburaya et al. disclosed that educational interventions could improve the scores of NOTSS (30). Improving the surgeons' non-technical skills could, in turn, lead to better surgery outcomes (34). In this context, the example behaviors listed in the NOTSS 
can be used as an educational package for surgeons and surgical residents. Training the surgeons regarding the necessary non-technical skills and incorporating some related courses in surgical residents' curriculum can be useful, as well. Considering the observations in the present study, setting some policies for the surgeons' full presence in the operating room can also be useful. Appropriate timing of surgeries can play an important role in the surgeons' full presence in the operating theater. Moreover, good supervision by the supervisor of the surgery ward is a key factor in implementing the standards. Using fixed team members for each surgeon can yet be another solution.

The low number of participants was unavoidable because of the low number of surgeons working at orthopedic wards. Furthermore, due to the existence of only two female surgeons, it was not possible to compare the scores of men and women surgeons.

\section{Conclusion}

The Persian version of NOTSS is a valid and reliable tool for assessing non-technical skills in Iranian surgeons. The studied surgeons had an acceptable level of non-technical skills, especially in the leadership domain. However, certain deficits were found in some behavioral elements; so, there is a need to pay more attention to improve other intrapersonal and cognitive skills. Educational, policymaking, and supervision interventions can be useful in improvement of nontechnical skills among surgeons.

\section{Acknowledgments}

The authors wish to express their sincere gratitude to the hospital officials and the surgeons who participated in this research. They would also like to acknowledge Ms. A. Keivanshekouh at the Research Improvement Center of Shiraz University of Medical Sciences for improving the use of English in the manuscript.

\section{Conflict of Interest}

Authors declared no conflict of interest.

\section{References}

1. de Vries EN, Ramrattan MA, Smorenburg SM, Gouma DJ, Boermeester MA. The incidence and nature of in-hospital adverse events: a systematic review. BMJ QualSafe. 2008;17(3):216-23. [DOI:10.1136/qshc.2007.023622]

2. Bogner MS. Misadventures in health care: inside stories: Psychology Press; 2003. [DOI:10.4324/9781410609038]

3. Flin R, O'Connor P. Safety at the sharp end: a guide to non-technical skills: CRC Press; 2017. [DOI:10.1201/9781315607467]

4. Flin R. Non-technical skills for anaesthetists, surgeons and scrub practitioners (ANTS, NOTSS and SPLINTS). The Healthcare Foundation. 2013:1-9.

5. Yule S, Flin R, Paterson-Brown S, Maran N. Nontechnical skills for surgeons in the operating room: a review of the literature. Surgery. 2006;139(2):140-9.

[DOI:10.1016/j.surg.2005.06.017]

6. Christian CK, Gustafson ML, Roth EM, et al. A prospective study of patient safety in the operating room. Surgery. 2006;139(2):159-73. [DOI:10.1016/j.surg.2005.07.037]

7. Garosi E, Kalantari R, Zanjirani Farahani A, Zuaktafi M, Hosseinzadeh Roknabadi E, Bakhshi E. Concerns about verbal communication in the operating room: A field study. Hum Factors.0018720819858274.

8. Gawande AA, Zinner MJ, Studdert DM, Brennan TA. Analysis of errors reported by surgeons at three teaching hospitals. Surgery. 2003;133(6):614-21. [DOI:10.1067/msy.2003.169]

9. Scotland I. Scottish Audit of Surgical Mortality Annual Report. 2013.

10. Smeltzer SC, Bare BG. Brunner \& Suddarth's textbook of medical-surgical nursing: JB Lippincott Philadelphia; 1992.

11. Agha RA, Fowler AJ, Sevdalis N. The role of nontechnical skills in surgery. Ann Med Surg. 2015;4(4):422-7.

[DOI:10.1016/j.amsu.2015.10.006]

12. Brunckhorst O, Shahid S, Aydin A, et al. The relationship between technical and nontechnical skills within a simulation-based ureteroscopy training environment. J Surg Educat. 2015;72(5):1039-44. [DOI:10.1016/].jsurg.2015.04.002] 
13. Greenberg CC, Regenbogen SE, Studdert DM, et al. Patterns of communication breakdowns resulting in injury to surgical patients. J Am College Surg. 2007;204(4):533-40. [DOI:10.1016/j.jamcollsurg.2007.01.010]

14. Moorthy K, Munz Y, Adams S, Pandey V, Darzi A. A human factors analysis of technical and team skills among surgical trainees during procedural simulations in a simulated operating theatre. Ann Surg. $2005 ; 242(5): 631$. [DOI:10.1097/01.sla.0000186298.79308.a8]

15. Jacklin R, Sevdalis N, Darzi A, Vincent C. Mapping surgical practice decision making: an interview study to evaluate decisions in surgical care. Am J Surg. 2008;195(5):689-96. [DOI:10.1016/j.amjsurg.2007.02.016]

16. Yule S, Flin R, Paterson-Brown S, Maran N, Rowley D. Development of a rating system for surgeons' non-technical skills. Medl Educat. 2006;40(11):1098-104. [DOI:10.1111/j.13652929.2006.02610.x]

17. Kalantari R, Zakerian SA, Mahmodi Majdabadi M, Zanjirani Farahani A, Meshkati M, Garosi E. Assessing the teamwork among surgical teams of hospitals affiliated to social security organizations in Tehran City. J Hospital. 2016;15(3):21-9.

18. Sexton JB, Makary MA, Tersigni AR, et al. Teamwork in the operating room: frontline perspectives among hospitals and operating room personnel. Anesthesiol. 2006;105(5):877-84. [DOI:10.1097/00000542-200611000-00006]

19. Rao R, Dumon KR, Neylan CJ, et al. Can simulated team tasks be used to improve nontechnical skills in the operating room? J Surg Educat. [DOI:10.1016/j.jsurg.2016.06.004]

20. Flin R, Yule S, Paterson-Brown S, Rowley D, Maran N. The non-technical skills for surgeons (NOTSS) system handbook v1. 2: Structuring observation, rating and feedback of surgeons behaviours in the operating theatre. Aberdeen, Scotland: University of Aberdeen Central Printing Service. 2006.

21. WHO guidelines for safe surgery: safe surgery saves lives: Sưu tầm; 2009.

22. Flin R, Youngson GG, Yule S. Enhancing surgical performance: A primer in non-technical skills: CRC press; 2015. [DOI:10.1201/b18702]

23. Wood TC, Raison N, Haldar S, et al. Training tools for nontechnical skills for surgeons-a systematic review. J Surg Educat. 2017;74(4):548-78.

[DOI:10.1016/j.jsurg.2016.11.017]
24. Jung JJ, Borkhoff CM, Jüni P, Grantcharov TP. Non-technical skills for surgeons (NOTSS): Critical appraisal of its measurement properties. Am J Surg. 2018;216(5):990-7. [DOI:10.1016/j.amjsurg.2018.02.021]

25. Yule S, Flin R, Maran N, Rowley D, Youngson G, Paterson-Brown S. Surgeons' non-technical skills in the operating room: reliability testing of the NOTSS behavior rating system. World J Surg. 2008;32(4):548-56. [DOI:10.1007/s00268-0079320-z]

26. Spanager L, Lyk-Jensen HT, Dieckmann P, Wettergren A, Rosenberg J, Ostergaard D. Customization of a tool to assess Danish surgeons' non-technical skills in the operating room. Dan Med J. 2012;59(11):A4526.

27. Wauben L, Dekker-van Doorn C, Van Wijngaarden J, et al. Discrepant perceptions of communication, teamwork and situation awareness among surgical team members. Int $\mathbf{J}$ Qual Health Care. 2011;23(2):159-66. [DOI:10.1093/intqhe/mzq079]

28. Mills P, Neily J, Dunn E. Teamwork and communication in surgical teams: implications for patient safety. J Am College Surg. 2008;206(1):107-12.

[DOI:10.1016/i.jamcollsurg.2007.06.281]

29. Williams RG, Silverman R, Schwind C, Fortune JB, Sutyak J, Horvath KD, et al. Surgeon information transfer and communication: factors affecting quality and efficiency of inpatient care. Ann Surg. 2007;245(2):159. [DOI:10.1097/01.sla.0000242709.28760.56]

30. Tsuburaya A, Soma T, Yoshikawa T, et al. Introduction of the non-technical skills for surgeons (NOTSS) system in a japanese Cancer center. Surg Today. 2016;46(12):1451-5. [DOI:10.1007/s00595-016-1322-8]

31. Yule S, Paterson-Brown S. Surgeons' nontechnical skills. Surg Clin. 2012;92(1):37-50. [DOI:10.1016/j.suc.2011.11.004]

32. Kalantari R, Farahani AZ, Garosi E, Badeli H, Jamali J. Translation and psychometric properties of the Persian version of oxford non-technical skills 2 system: Assessment of surgical teams' non-technical skills in orthopedic surgery wards. Arch Bone Joint Surg. 2019;7(2):173.

33. Dedy NJ, Zevin B, Bonrath EM, Grantcharov TP. Non-technical skills of surgery residents: does experiential learning lead to competence? J Am College Surg. 2013;217(3):S116. [DOI:10.1016/j.jamcollsurg.2013.07.266] 
34. Ounounou E, Aydin A, Brunckhorst O, Khan MS, Dasgupta P, Ahmed K. Nontechnical skills in surgery: A systematic review of current training modalities. J Surg Educat. 2019;76(1):14-24. [DOI:10.1016/j.jsurg.2018.05.017]

\section{How to Cite This Article:}

Hasanshahi M, Kalantari R, Zamanian Z, Gheysari S, Bakhshi E. Assessment of Non-Technical Skills in Iranian

Orthopedic Surgeons: An Observational Study. J Adv Med Biomed Res. 2020; 28 (129) :191-197

\section{Download citation:}

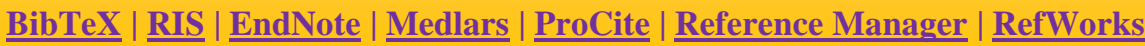

\section{Send citation to:}

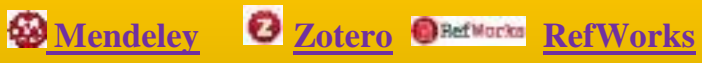

